\title{
ПРАКТИЧЕСКИЕ РЕКОМЕНДАЦИИ ПО ПРОФИЛАКТИКЕ И ЛЕЧЕНИЮ ТОШНОТЫ И РВОТЫ У ОНКОЛОГИЧЕСКИХ БОЛЬНЫХ
}

Коллектив авторов: Владимирова Л.Ю., Гладков О.А., Королева И.А., Румянцев А.А., Семиглазова Т.Ю., Трякин А.А.

DOI: $10.18027 / 2224-5057-2019-9-3 s 2-37$

Ключевые слова: поддерживающая терапия, антиэметики, тошнота и рвота при химиотерапии, профилактика тошноты и рвоты

Тошнота и рвота являются одним из наиболее частых побочных эффектов химиотерапии (XT) и существенно ухудшают качество жизни пациентов.

\section{1. КЛАССИФИКАЦИЯ}

\section{1. Типы тошноты и рвоты}

- Острая рвота развивается в первые 24 ч после химиотерапии, отличается высокой интенсивностью, редко сопровождается тошнотой.

- Отсроченная рвота развивается на 2-5-е сутки после начала химиотерапии, менее интенсивна, чем острая, и, как правило, сопровождается постоянной тошнотой.

- Условно-рефлекторная рвота представляет собой классический условный рефлекс на химиотерапию и / или сопутствующие ей манипуляции и окружающую обстановку. Формируется в тех случаях, когда противоопухолевая терапия сопровождается тошнотой и рвотой. Риск её развития увеличивается пропорционально числу проведённых курсов и может сохраняться в течение длительного времени после окончания химиотерапии.

- Неконтролируемая (breakthrough - «прорывная») тошнота и рвота развивается на фоне адекватной антиэметической профилактики и требует дополнительной коррекции.

- Рефрактерная рвота возникает на последующих циклах химиотерапии при неэффективности противорвотной профилактики и / или препаратов резерва на предыдущих курсах лечения.

Цитирование: Владимирова Л.Ю., Гладков О.А., Королева И.А., Румянцев А.А., Семиглазова Т.Ю., Трякин А.А. Практические рекомендации по профилактике и лечению тошноты и рвоты у онкологических больных. Злокачественные опухоли: Практические рекомендачии RUSSCO \#3s2, 2020 (том 10).37 


\section{2. Эметогенный потенциал противоопухолевого препарата}

Под уровнем эметогенности понимается риск развития рвоты у больных, получающих тот или иной цитостатик в монорежиме без противорвотной терапии. Классификация противоопухолевых препаратов в зависимости от уровня эметогенности по рекомендациям MASCC/ESMO представлена в табл. 1.

Эметогенный потенциал препаратов для внутривенного введения определяется чаще всего для режима однодневного введения, то есть устанавливают эметогенность разовой дозы. В основу эметогенной классификации таблетированных препаратов положен учёт эметогенности полного курса лечения.

Эметогенность комбинированного режима химиотерапии определяется, как правило, препаратом, обладающим наибольшим эметогенным потенциалом. Это положение является абсолютно верным для режимов, включающих высокоэметогенные цитостатики.

Комбинация умеренно эметогенных противоопухолевых препаратов может повышать эметогенность режима в целом.

Таблица 1. Эметогенный потенциал противоопухолевых препаратов (в монотерапии), схем и режимов. Рекомендации MASCC/ESMO 2016 г., ASCO 2017 г.

\begin{tabular}{|c|c|c|}
\hline Уровень эметогенности & $\begin{array}{l}\text { Противоопухолевые препараты для } \\
\text { внутривенного введения }\end{array}$ & $\begin{array}{l}\text { Противоопухолевые препараты } \\
\text { для приёма внутрь }\end{array}$ \\
\hline $\begin{array}{l}\text { Высокий } \\
\text { (рвота у } 90 \% \\
\text { больных и более) }\end{array}$ & 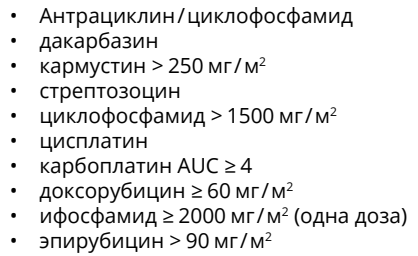 & $\begin{array}{l}\text { - Гексаметилмеламин } \\
\text { - прокарбазин }\end{array}$ \\
\hline $\begin{array}{l}\text { Умеренный } \\
\text { (рвота у 30-90\% } \\
\text { больных) }\end{array}$ & 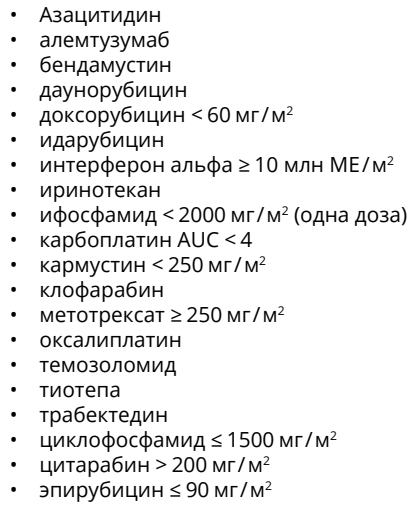 & 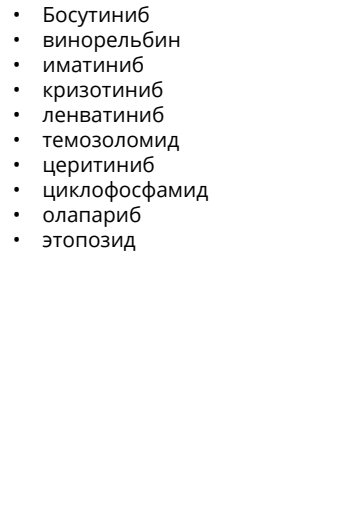 \\
\hline
\end{tabular}




\begin{tabular}{|c|c|c|}
\hline Уровень эметогенности & $\begin{array}{l}\text { Противоопухолевые препараты для } \\
\text { внутривенного введения }\end{array}$ & $\begin{array}{l}\text { Противоопухолевые препараты } \\
\text { для приёма внутрь }\end{array}$ \\
\hline $\begin{array}{l}\text { Низкий } \\
\text { (рвота у 10-30\%) }\end{array}$ & 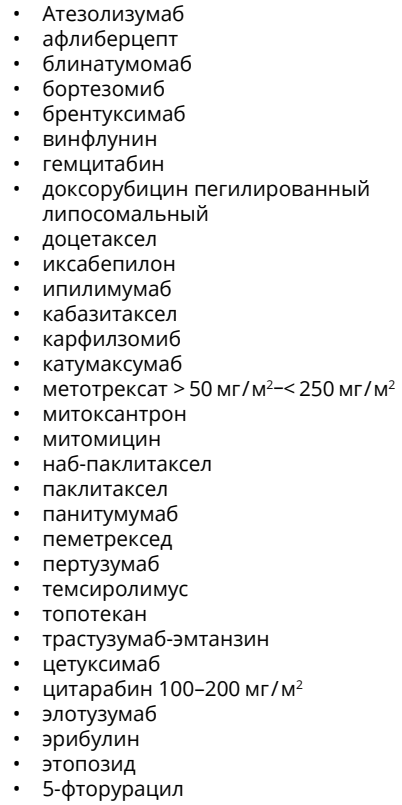 & 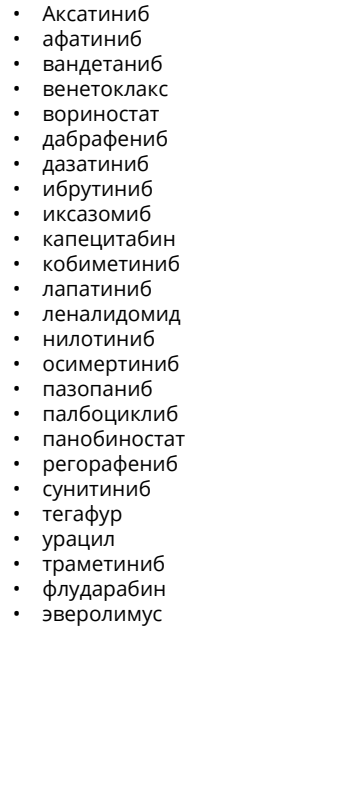 \\
\hline $\begin{array}{l}\text { Минимальный } \\
(<10 \% \text { больных) }\end{array}$ & $\begin{array}{l}\text { - Бевацизумаб } \\
\text { - блеомицин } \\
\text { - бусульфан } \\
\text { - винбластин } \\
\text { - винкристин } \\
\text { - динорельбин } \\
\text { - кладатумумаб } \\
\text { - ниволумаб } \\
\text { - обинутузумаб } \\
\text { - офатумумаб } \\
\text { - пембролизумаб } \\
\text { - пиксантрон } \\
\text { - рамуцирумаб } \\
\text { - ритуксимаб } \\
\text { - трастузумаб } \\
\text { - флударабин }\end{array}$ & 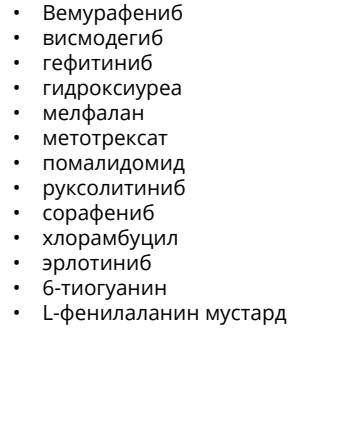 \\
\hline
\end{tabular}

\section{2. ЛЕЧЕНИЕ}

\section{1. Противорвотные препараты}

Основные препараты для профилактики тошноты и рвоты, а также особенности их использования представлены в табл. 2. 
Таблица 2. Группы препаратов и препараты для лечения тошноты и рвоты

\begin{tabular}{|c|c|}
\hline Группы препаратов/препараты & Особенности применения \\
\hline $\begin{array}{l}\text { Антагонисты 5-НТ3-рецепторов: } \\
\text { ондансетрон, гранисетрон, тропи- } \\
\text { сетрон, палоносетрон }\end{array}$ & $\begin{array}{l}\text { У пациентов с врождённым долгосрочным QТ-синдромом следует } \\
\text { избегать применения препаратов первого поколения } \\
\text { 5-НТЗ-антагонистов, за исключением палоносетрона, который не } \\
\text { оказывает влияния на параметры ЭКГ (интервал QТ). Рекомендуется } \\
\text { мониторинг ЭКГ у больных с признаками кардиальных нарушений, } \\
\text { включая сердечную недостаточность, брадиаритмию. Также может } \\
\text { быть целесообразно оценить концентрацию калия и магния в плаз- } \\
\text { ме крови и провести корректирующую терапию в случае выявления } \\
\text { клинически значимых отклонений. } \\
\text { Палоносетрон является препаратом последнего поколения, имеет } \\
\text { самый длительный период полувыведения (до } 40 \text { часов), в } 30 \text { раз } \\
\text { более сильное сродство к 5НТЗ рецепторам, чем препараты первого } \\
\text { поколения. Применяется однократно с 1-го дня 1-го цикла. При мно- } \\
\text { годневных курсах химиотерапии возможно применение препарата } \\
\text { через день - при многодневных введениях высокоэметогенных } \\
\text { химиопрепаратов. }\end{array}$ \\
\hline $\begin{array}{l}\text { Глюкокортикостероиды: декса- } \\
\text { метазон }\end{array}$ & $\begin{array}{l}\text { Дексаметазон не назначают дополнительно при проведении режи- } \\
\text { мов XT, уже содержащих дексаметазон. Дексаметазон противопока- } \\
\text { зан при терапии интерлейкином-2 и интерферонами. }\end{array}$ \\
\hline $\begin{array}{l}\text { Антагонисты NK1-рецепторов: } \\
\text { апрепи- тант, фосапрепитант, } \\
\text { нетупитант }\end{array}$ & $\begin{array}{l}\text { Являются умеренным ингибитором и индуктором СҮРЗА4, что } \\
\text { необходимо учитывать при одновременном использовании } \\
\text { препаратов, метаболизирующихся этой же системой. Например, } \\
\text { апрепитант снижает эффективность гормональных контрацептивов, } \\
\text { в связи с чем необходимо использование альтернативных методов } \\
\text { контрацепции. Апрепитант повышает концентрацию кортикосте- } \\
\text { роидов. В комбинации с апрепитантом доза дексамета- зона должна } \\
\text { быть снижена приблизительно на 50\%. У пациентов, получающих } \\
\text { антивитамины К (варфарин), необходимо дополнительно, до } 2 \text { раз } \\
\text { в неделю, контролировать уровень МНО (международного нормали- } \\
\text { зованного отношения). }\end{array}$ \\
\hline $\begin{array}{l}\text { Блокаторы рецепторов допами- } \\
\text { на: бензамиды (метоклопрамид, } \\
\text { итоприд), фенотиазины (хлорпро- } \\
\text { мазин или ами- назин, прометазин, } \\
\text { метопемазин), бути- рофеноны } \\
\text { (дроперидол, галоперидол), бензо- } \\
\text { диазепины (диазепам, лоразепам, } \\
\text { альпрозолам) }\end{array}$ & Обладают седативными и анксиолитическим свойствами. \\
\hline Нейролептики: оланзапин & $\begin{array}{l}\text { Обладает аффинностью к множеству рецепторов в центральной } \\
\text { нервной системе, участвующих в патогенезе тошноты и рвоты на } \\
\text { фоне проведения химиотерапии, включая 5-НT3 рецепторы, Н1-ги- } \\
\text { стаминорецепторы, D1 - 4-рецепторы. Может вызвать седатацию, } \\
\text { особенно у пожилых. }\end{array}$ \\
\hline
\end{tabular}

\section{3. ПРОФИЛАКТИКА}

\section{1. Алгоритм профилактики и терапии тошноты и рвоты}

- Определить эметогенный потенциал назначенного режима ХТ (табл. 1).

- Назначить профилактическую терапию, исходя из эметогенности режима XT, начиная с первого курса.

- Назначить лечение в случае развития тошноты и рвоты на фоне профилактической терапии. 
- В случае развития тошноты и рвоты внести изменения в профилактическую терапию на последующих циклах ХT.

Критерием эффективности противорвотной терапии является полное отсутствие (полный контроль) рвоты и тошноты в течение острого и отсроченного периода.

\section{2. Профилактика острой и отсроченной тошноты и рвоты}

\subsection{1. Профилактика острой и отсроченной тошноты и рвоты при высокоэметогенной однодневной химиотерапии}

- Профилактика тошноты и рвоты, возникающей при проведении химиотерапии с высокоэметогенным потенциалом, должна начинаться до начала химиотерапии и проводиться не менее 3 дней после проведения химиотерапии. В настоящее время наиболее эффективной антиэметическими режимами являются комбинации противорвотных препаратов, включающие:

1. Антагонист NK1-рецепторов + оланзапин + антагонист рецепторов серотонина (5-НT3) + дексаметазон;

2. антагонист NK1-рецепторов + антагонист рецепторов серотонина (5-HT3) + дексаметазон;

3. Оланзапин + антагонист рецепторов серотонина (5-HT3) + дексаметазон

- Для пациентов с низким индивидуальным риском развития тошноты и рвоты на фоне химиотерапии возможно применение двухкомпонентных режимов профилактики ТиР (ондансетрон/дексаметазон и прочее) при обязательной эскалации противорвотной терапии при развитии ТиР после проведения 1 курса лечения.

- Добавление бензодиазепинов и блокаторов Н2-гистаминорецепторов или ингибиторов протонной помпы проводится по показаниям на усмотрение лечащего врача (табл. 3).

Таблица 3. Профилактика рвоты и тошноты при однодневной высокоэметогенной химиотерапии

\begin{tabular}{|c|c|c|c|c|c|}
\hline Группа & Препарат & День 1 & День 2 & День 3 & День 4 \\
\hline \multirow[t]{2}{*}{$\begin{array}{l}\text { Блокаторы } \\
\text { NK1-рецеп- } \\
\text { торов }\end{array}$} & $\begin{array}{l}\text { Апрепитант } \\
\text { или }\end{array}$ & $\begin{array}{l}125 \text { мг внутрь } \\
1 \text { раз в день за } \\
60 \text { мин до XТ }\end{array}$ & $\begin{array}{l}80 \text { мг внутрь } \\
1 \text { раз утром }\end{array}$ & $\begin{array}{l}80 \text { мг внутрь } \\
1 \text { раз утром }\end{array}$ & - \\
\hline & Фосапрепитант & $\begin{array}{l}150 \text { мг в/в, } \\
\text { капельно } \\
\text { (однократно) за } \\
30 \text { минут до ХТ }\end{array}$ & - & - & - \\
\hline Нейролептики & Оланзапин & $\begin{array}{l}5 \text { мг внутрь } \\
\text { не менее, чем } \\
\text { за } 1 \text { час до ХT } \\
\text { или накануне } \\
\text { вечером* }^{*}\end{array}$ & $\begin{array}{l}5 \text { мг внутрь } \\
1 \text { раз в день }\end{array}$ & $\begin{array}{l}5 \text { мг внутрь } \\
1 \text { раз в день }\end{array}$ & $\begin{array}{l}5 \text { мг внутрь } \\
1 \text { раз в день }\end{array}$ \\
\hline
\end{tabular}




\begin{tabular}{|c|c|c|c|c|c|}
\hline Группа & Препарат & День 1 & День 2 & День 3 & День 4 \\
\hline \multirow[t]{5}{*}{$\begin{array}{l}\text { Блокатор } \\
\text { 5-НТ3-рецеп- } \\
\text { торов }\end{array}$} & $\begin{array}{l}\text { Палоносетрон } \\
\text { или }\end{array}$ & $\begin{array}{l}\text { 0,25 мг в/в (од- } \\
\text { нократно) за } \\
30-60 \text { минут } \\
\text { до ХТ }\end{array}$ & - & - & - \\
\hline & $\begin{array}{l}\text { Ондансетрон } \\
\text { или }\end{array}$ & $\begin{array}{l}\text { 8-16 мг в/в или } \\
\text { 8-16 мг внутрь } \\
\text { за 30-60 минут } \\
\text { до ХТ }\end{array}$ & - & - & - \\
\hline & $\begin{array}{l}\text { Гранисетрон } \\
\text { или }\end{array}$ & $\begin{array}{l}1-3 \text { мг в/в или } \\
2 \text { мг внутрь за } \\
30-60 \text { ми нут } \\
\text { до ХТ }\end{array}$ & - & - & - \\
\hline & Трописетрон & $\begin{array}{l}5 \text { мг в/в или } \\
\text { внутрь за } \\
\text { 30-60 минут } \\
\text { до ХТ }\end{array}$ & - & - & - \\
\hline & $\begin{array}{l}\text { Комбинирован- } \\
\text { ный блокатор } \\
\text { NK1-рецепто- } \\
\text { ров и 5-НT3-ре- } \\
\text { цепторов }\end{array}$ & $\begin{array}{l}\text { Нетупитант+ } \\
\text { Палоносетрон }\end{array}$ & $300 \mathrm{Mr}+0,5 \mathrm{Mr}$ & & \\
\hline $\begin{array}{l}\text { Глюкокортико- } \\
\text { стероиды }\end{array}$ & Дексаметазон & $\begin{array}{l}12 \text { мг внутри- } \\
\text { венно } 1 \text { раз за } \\
30-60 \text { минут } \\
\text { до ХТ }\end{array}$ & $\begin{array}{l}8 \text { мг внутрь } \\
\text { или в/м } 2 \text { раза } \\
\text { в день }\end{array}$ & $\begin{array}{l}8 \text { мг внутрь } \\
\text { или в/м } 2 \text { раза } \\
\text { в день }\end{array}$ & $\begin{array}{l}8 \text { мг внутрь } \\
\text { или в/м } 2 \text { раза } \\
\text { в день, до } \\
5 \text { дней }\end{array}$ \\
\hline $\begin{array}{l} \pm \text { Бензодиазе- } \\
\text { пины }\end{array}$ & Лоразепам & \multicolumn{4}{|c|}{$\begin{array}{l}\text { 0,5-2 мг внутрь или внутримышечно/внутривенно, при этом разовая } \\
\text { доза составляет } 50 \text { мкг/кг каждые 4-6 ч. Максимальная суточная доза } \\
\text { при приеме внутрь составляет } 10 \text { мг. При внутримышечном или вну- } \\
\text { тривенном введении разовая доза - } 4 \text { мг. }\end{array}$} \\
\hline
\end{tabular}

* При недостаточном эффекте противорвотной терапии и удовлетворительной переносимости препарата возможно увеличение дозы до 10 мг/сутки

Рекомендованной дозой ондансетрона для пациентов моложе 60 лет является 8 мг внутривенно и 16 мг перорально (в контролируемых исследованиях 8 мг перорально 2 раза в сутки). Для пациентов старше 60 лет рекомендованная доза ондансетрона - 8 мг внутривенно и 8 мг перорально.

При использовании фосапрепитанта в 1-й день, дексаметазон на 2-й-5-й дни может не назначаться или использоваться в дозе 8 мг 1 раз в день.

Рандомизированные исследования показали, что на фоне применения палоносетрона доза дексаметазона может быть уменьшена до 8 мг в 1-й день профилактики и не использоваться на 2-й и 3-й дни.

\subsection{2. Профилактика острой и отсроченной тошноты и рвоты при умеренноэметогенной однодневной химиотерапии}

- Профилактика тошноты и рвоты, возникающей при проведении химиотерапии с умеренно эметогенным потенциалом, должна начинаться до начала химиотерапии и проводиться не менее 3 дней после проведения 
химиотерапии. В настоящее время наиболее эффективной антиэметической комбинацией является комбинация противорвотных препаратов, включающая (табл. 4): антагонист рецепторов серотонина (5-НT3) + дексаметазон.

- При наличии других индивидуальных факторов риска развития тошноты и рвоты рекомендуется рассмотреть возможность назначения трехкомпонентных режимов профилактики тошноты и рвоты с использованием апрепитанта / фосапрепитанта или оланзапина

Таблица 4. Профилактика рвоты и тошноты при умеренно эметогенной однодневной химиотерапии

\begin{tabular}{|c|c|c|c|c|}
\hline Группа & Препарат & День 1 & День 2 & День 3 \\
\hline \multirow[t]{4}{*}{$\begin{array}{l}\text { Блокатор } \\
\text { 5-НТ3-рецепторов }\end{array}$} & $\begin{array}{l}\text { Палоносетрон } \\
\text { или }\end{array}$ & $\begin{array}{l}\text { 0,25 мг в/в (однократно) } \\
\text { за 30-60 минут до ХТ }\end{array}$ & - & - \\
\hline & $\begin{array}{l}\text { ондансетрон } \\
\text { или }\end{array}$ & $\begin{array}{l}8 \text { мг в/в или 8-16 мг внутрь } \\
\text { за 30-60 минут до ХТ }\end{array}$ & - & - \\
\hline & $\begin{array}{l}\text { гранисетрон } \\
\text { или }\end{array}$ & $\begin{array}{l}\text { 1-3 мг в/в или } 2 \text { мг внутрь } \\
\text { за 30-60 минут до ХТ }\end{array}$ & - & - \\
\hline & трописетрон & $\begin{array}{l}5 \text { мг в/в или внутрь за } \\
30-60 \text { минут до XТ }\end{array}$ & - & - \\
\hline Глюкокортикоиды & Дексаметазон & $\begin{array}{l}\text { 8-12 мг внутривенно или } \\
\text { внутрь за 30-60 минут до XТ }\end{array}$ & $\begin{array}{l}8 \text { мг внутрь или } \\
\text { В/м }\end{array}$ & $\begin{array}{l}8 \text { мг внутрь или } \\
\text { в/м }\end{array}$ \\
\hline \pm Бензодиазепины & Лоразепам & \multicolumn{3}{|c|}{ 0,5-2,0 мг внутрь или внутривенно каждые 4-6 ч. } \\
\hline
\end{tabular}

Рекомендованной дозой ондансетрона для пациентов моложе 60 лет является 8 мг внутривенно или 16 мг перорально (в контролируемых исследованиях - 8 мг п/о 2 раза в сутки). У пациентов старше 60 лет рекомендованная доза ондансетрона - 8 мг внутривенно или 8 мг перорально.

Рандомизированные исследования показали, что на фоне применения палоносетрона доза дексаметазона может быть уменьшена до 8 мг в 1-й день профилактики и не использоваться на 2-й и 3-й дни.

Назначение дексаметазона на 2-й-3-й дни производится при наличии тошноты, рвоты или на усмотрение лечащего врача. Исключение составляют схемы, содержащие карбоплатин, АC, BEP, при которых дексаметазон назначается, как при ВэХТ.

\subsection{3. Профилактика острой и отсроченной тошноты и рвоты при низкоэметогенной однодневной химиотерапии}

Для профилактики тошноты и рвоты при химиотерапии с низкой эметогенностью следует использовать только один из препаратов: дексаметазон, антагонист 5-НТ3-рецепторов или антагонист рецепторов допамина (например, метоклопрамид) (табл. 5). 
Таблица 5. Профилактика острой и отсроченной тошноты и рвоты при низкоэметогенной однодневной химиотерапии

\begin{tabular}{|l|l|}
\hline Группа & Препарат \\
\hline Глюкокортикоиды & Дексаметазон 8 мг внутрь или внутривенно в день 1 \\
\hline \multicolumn{1}{|l|}{ или } \\
\hline $\begin{array}{l}\text { Блокатор } \\
\text { 5-НТ3-рецепторов }\end{array}$ & $\begin{array}{l}\text { Ондансетрон внутрь, в свечах или внутривенно, однократно, за 30-60 мин до ХT } \\
\text { или гранисетрон 1-3 мг в/в или 2 мг внутрь за 30-60 минут до ХТ } \\
\text { или трописетрон 5 мг в/в или внутрь за 30-60 минут до ХТ } \\
\text { или палоносетрон 0,25 мг в/в за 30-60 минут до ХТ }\end{array}$ \\
\hline \multicolumn{1}{|l|}{\begin{tabular}{l} 
или \\
\hline $\begin{array}{l}\text { Блокатор рецепто- } \\
\text { ров допамина }\end{array}$
\end{tabular}} & Метоклопрамид 10-40 мг внутрь или внутривенно за 30-60 мин до ХТ \\
\hline
\end{tabular}

\subsection{4. Профилактика тошноты и рвоты при минимально эметогенной однодневной химиотерапии}

Профилактика не требуется.

\subsection{5. Профилактика тошноты и рвоты при многодневной химиотерапии с высоким риском эметогенности}

- У пациентов, получающих многодневную химиотерапию, граница между острой и отсроченной тошнотой и рвотой стирается, перекрываются острый и отсроченный периоды, при этом имеет значение как индивидуальная эметогенность цитостатиков, так и их сочетания. Риск развития отсроченных эметических реакций зависит как от эметогенности режима, так и от эметогенного потенциала последнего введённого цитостатика. Поэтому профилактика тошноты и рвоты должна проводиться с 1-го дня цикла ХТ и ещё в течение 2-3 дней после его окончания. Антиэметики назначают ежедневно (исключение составляет палоносетрон, назначаемый через день) на основе эметогенного потенциала цитостатиков, вводимых в тот или иной день. Выбор противорвотной комбинации осуществляется на основе препарата, обладающего наибольшей эметогенностью.

- Антагонист 5-НT3-рецепторов должен быть назначен до первого введения умеренно- или высокоэметогенного препарата. По данным клинических исследований, при многодневных курсах химиотерапии возможно введение палоносетрона через день, то есть рекомендуемая доза составляет 0,25 мг в 1-й, 3-й, 5-й дни химиотерапии.

Дексаметазон должен быть назначен в 1-й день для умеренно и высокоэметогенных препаратов внутрь или внутривенно, затем через 2-3 дня после окончания ХT. Дексаметазон не добавляют к режимам, уже содержащим дексаметазон (при лечении онкогематологических заболеваний). 
Апрепитант может быть назначен при высокоэметогенной многодневной химиотерапии в стандартном режиме ИЛИ в дозе 125 мг в 1 день и 80 мг в 2-5 дни или в режиме 125 мг внутрь день 3, 80 мг внутрь в дни 4-7.

Возможно применение оланзапина в дозе 5-10 мг внутрь в дни введения высокоэметогенных препаратов и до трех дней после завершения химиотерапии.

\subsection{6. Профилактика тошноты и рвоты, возникающей при проведении лучевой терапии}

Профилактика тошноты и рвоты, возникающей на фоне лучевой терапии, проводится с учётом зоны облучения (табл. 6). При недостаточной эффективности антагонистов 5-НТ3-рецепторов в монотерапии возможно присоединение глюкокортикоидов. Предпочтительно использовать препараты в пероральных формах.

Таблица 6. Профилактика тошноты и рвоты, возникающей при проведении лучевой терапии

\begin{tabular}{|c|c|c|}
\hline Зона облучения & Группа & Препараты \\
\hline \multirow[t]{2}{*}{$\begin{array}{l}\text { Область верхней части } \\
\text { живота, краниоспиналь- } \\
\text { ной зоны }\end{array}$} & Антагонисты 5-НТ3-рецепторов & $\begin{array}{l}\text { Палоносетрон 0,25 мг внутривенно } \\
\text { однократно в день (через день) или он- } \\
\text { дансетрон 16-24 мг внутрь или ректально } \\
\text { (предпочтительно) или внутривенно, или } \\
\text { внутримышечно в день или гранисетрон } \\
2 \text { мг внутрь (предпочтительно) или 1-3 мг } \\
\text { внутривенно или внутримышечно в день } \\
\text { или трописетрон } 5 \text { мг внутрь (предпочти- } \\
\text { тельно) или внутривенно или внутримы- } \\
\text { шечно в день }\end{array}$ \\
\hline & $\begin{array}{l}\text { Глюкокортикоиды (в сочетании } \\
\text { с антагонистами 5-НТЗ-рецепто- } \\
\text { ров при недостаточной их эффек- } \\
\text { тивности в монотерапии) }\end{array}$ & $\begin{array}{l}\text { Дексаметазон } 4 \text { мг внутрь (предпочтитель- } \\
\text { но) внутривенно или внутримышечно. } \\
\text { Препараты применяют за 30-60 мин до } \\
\text { облучения }\end{array}$ \\
\hline $\begin{array}{l}\text { 1. Облучение черепа, } \\
\text { области головы - шеи, } \\
\text { нижней части грудной } \\
\text { клетки, таза } \\
\text { 2. Области молочных } \\
\text { желез, конечностей }\end{array}$ & \multicolumn{2}{|c|}{$\begin{array}{l}\text { У данной группы пациентов проведения профилактики тошноты и рвоты } \\
\text { не требуется. В случае наличия тошноты и рвоты можно назначить любой } \\
\text { препарат из группы антагонистов 5-НТ3-рецепторов. Препараты применяют за } \\
\text { 30-60 мин до облучения. Предпочтительны пероральные формы. }\end{array}$} \\
\hline
\end{tabular}

\subsection{7. Проведение профилактики тошноты и рвоты при сочетании химиотерапии и лучевой терапии}

При проведении лучевого воздействия на фоне химиотерапии при антиэметической профилактике рекомендуется:

- Определять эметогенный потенциал по максимально эметогенному воздействию (по зоне лучевой нагрузки или по цитостатику);

- При наличии 3 или более факторов риска (женский пол, малый приём алкоголя, страх перед лечением, возраст моложе 55 лет и тошнота и рвота в анамнезе) эметогенность может быть поднята на одну степень выше. 


\section{3. Терапия неконтролируемой тошноты и рвоты}

При развитии прорывной тошноты и рвоты предлагается следующий алгоритм действий:

\subsection{1. Дополнительная фармакологическая коррекция тошноты и рвоты}

Могут использоваться следующие лекарственные препараты:

- Оланзапин 10 мг внутрь 1 раз в сутки внутрь в течение 3 дней (предпочтельно);

- Дексаметазон 12 мг в/в или внутрь;

- Метоклопрамид 10-20 мг в/в или внутрь каждые 4-6 часов;

- Использование «дополнительной» дозы антагониста 5-НТ-3 рецепторов: ондансетрон 8-16 мг в/в или 16-24 мг внутрь ИлИ гранисетрон 1 мг в/в или 1-2 мг внутрь ИЛИ палоносетрон 0,25 мг в/в 1

- Лоразепам по 0,5-2 мг каждые 4-6 часов;

- Аминазин 25 мг внутрь каждые 6 часов ИлИ галоперидол 1-2 мг внутрь каждые 4-6 часов.

Оптимальный подход к фармакотерапии «прорывной» тошноты и рвоты на фоне химиотерапии в настоящее время не определен. По данным рандомизированного исследования оланзапин достоверно превосходит метоклопрамид при развитии данного осложнения в связи с чем применение данного препарата является предпочтительной опцией.

Результаты небольшого рандомизированного исследования показали эффективность замены ондансетрона на гранисетрон у пациентов с недостаточным эффектом антиэметогенной терапии при проведении последующих курсов химиотерапии, что указывает на целесообразность применения этого препарата у пациентов, с развитием «прорывной» ТиР на фоне применения ондансетрона. Кроме того, антагонисты 5-НТ3-рецепторов имеют различный метаболизм в печени, у ряда пациентов с так называемым сверхбыстрым метаболическим фенотипом может наблюдаться ускоренное выведение препаратов из организма (например, трописетрона). В данной ситуации также целесообразна замена 5-НТ3-блокатора на другой представитель данного класса.

В случае недостаточного контроля рвоты и отсутствии дополнительных причин развития тошноты и рвоты (см. ниже) возможно применение нескольких препаратов из перечисленного списка - исходя из индивидуального соотношения пользы и риска их применения. В случае развития рвоты предпочтительным может являться парентеральный путь введения препаратов либо введение в ректальных свечах по сравнению с их пероральным назначением (при доступности соответствующих лекарственных форм).

\footnotetext{
1 Препарат может применяться не чаще, чем через день (1 раз в 48 часов)
} 


\subsection{2. Оценка наличия других причин развития тошноты и рвоты на фоне проводимого лечения}

- Обструкция или парез кишечника (винкристин);

- Метастатическое поражение центральной нервной системы, лептоменингеальный канцероматоз;

- Гиперкальциемия, гипонатриемия, гипергликемия;

- Уремия;

- Назначение опиатов;

- Психофизиологические (рвота ожидания).

В случае подозрения или выявления какой-либо из вышеперечисленных причин следует провести их коррекцию.

В случае развития выраженной рвоты на фоне химиотерапии следует проводить коррекцию гиповолемии и электролитных нарушений вне зависимости от причины, вызвавшей рвоту.

При наличии явлений диспепсии целесообразно применение Н2-гистаминоблокаторов или ингибиторов протонной помпы.

\subsection{3. Принципы профилактики и лечения «прорывной» тошноты и рвоты}

При проведении последующих курсов химиотерапии у пациентов с отмеченным развитием «прорывной» тошноты и рвоты следует соблюдать ряд принципов профилактики и лечения тошноты и рвоты:

- Провести эскалацию режима противорвотной терапии до следующего уровня эметогенности. Пациентам, получавшим трехкомпонентные режимы профилактики тошноты и рвоты следует назначать четырехкомпонентные режимы;

- При возникновении неконтролируемой тошноты и рвоты на фоне четырехкомпонентной противорвотной схемы стандартного подхода нет. Следует рассмотреть возможность профилактического применения подхода, который был использован для лечения тошноты и рвоты;

- Назначать антиэметики строго по часам, а не только при появлении тошноты или рвоты.

\section{4. Профилактика и терапия условно-рефлекторной рвоты}

Для профилактики и лечения условно-рефлекторной тошноты и рвоты применяются:

- Оптимальная профилактика тошноты и рвоты при каждом цикле XТ;

- Бензодиазепины: лоразепам по 0,5-2 мг на ночь накануне и утром перед химиотерапией;

- Психотерапия, гипноз, акупунктура, арт-терапия, системная десенсибилизация. 